\title{
"Imperfect Love": The Effect of Borderline Personality Disorder Symptoms and Relationship Commitment on Relationship Satisfaction
}

\author{
Grace White $^{1^{*}}$, Ph.D, Roberta Napoleon ${ }^{2}$, B. A.
}

1*Associate Lecturer, Department of Psychology, University of Central Florida, United States. ${ }^{2}$ Clinical Masters Student, Department of Psychology, University of Central Florida,United States.

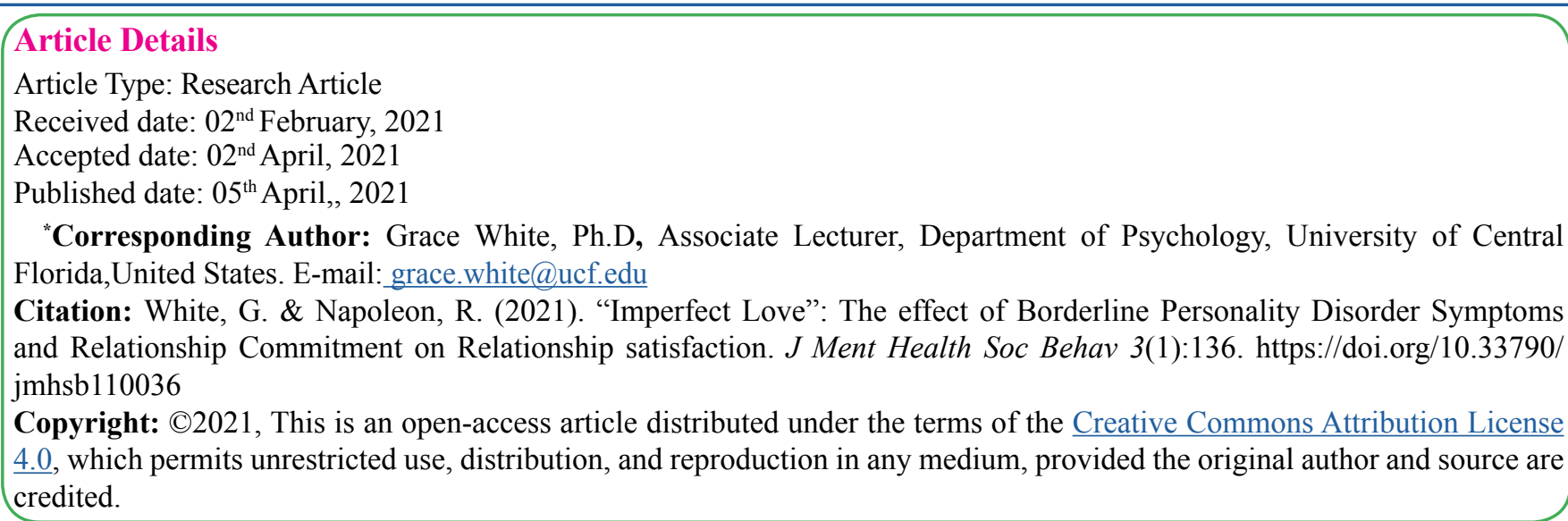

\section{Abstract}

This study aimed to determine the connection between borderline personality disorder (BPD) symptom-endorsement and relationship satisfaction. Individuals in romantic relationships often report higher levels of overall life satisfaction and functioning. However, the experience of emotional distress or disability may impair one's ability to benefit from the protective effects of a romantic dyad. Therefore, understanding the impact that individual mental health may have on perceptions of satisfaction in romantic relationships may be of importance. Additionally, the role that relationship type, such as committed vs. non-committed relationships, can have on assessments of satisfaction for individuals who experience BPD symptoms is of interest. 433 participants self-reported their experiences with BPD symptoms using the Borderline Symptoms List 23 [1] and relationship type (committed vs. non-committed). The Relationship Satisfaction Assessment [2] was completed to determine individuals' happiness and fulfillment within their interpersonal relationships. Findings indicated that endorsement of BPD symptoms had a significant negative association with self-reported relationship satisfaction. Relationship type was also a significant indicator of relationship satisfaction. Thus, those in committed relationships reported higher levels of overall satisfaction than those in non-committed relationships. This research suggests that both the experience of certain mental health issues and commitment to a relationship may determine overall feelings of fulfillment in romantic relationships. Additional research is necessary to replicate and explore the impact of this association.

Keywords: Mental health, clinical syndromes, interpersonal relations, intimate dyads

\section{Introduction}

Borderline Personality Disorder (BPD) ${ }^{1}$ is a mental disorder that is multifaceted. Those with the disorder frequently experience the inability to maintain stable control over impulses, emotions, selfimage, and dyadic interactions [3]. This instability can present itself as different characteristics, such as mood swings and depression, which can be detrimental to interpersonal relationships [4]. In an examination of borderline and avoidant personality disorders, borderline personality disorder was found to specifically predict romantic relationship dysfunction [5]. However, little attention has been given to specific features of interpersonal relationships, such as whether the relationship is committed or non-committed, when examining the experiences and functioning of individuals who may have sub-clinical levels of the syndrome. Thus, having a broader understanding of the links between symptoms of BPD and specific relationship processes may have practical implications for both informal and formal treatments of the disorder and for improving relationship dysfunction for those who have yet to be diagnosed with the condition.

Data from the National Institute on Mental Health (2019) suggests that there has been a rise in individuals seeking out therapeutic and pharmaceutical treatments for symptoms of a variety of psychological ailments. Although this increase in formal therapies is important, past research suggests that people may also benefit psychologically and physically from informal remedies, such as the social support received within interpersonal relationships [6]. In a meta-analysis, Henderson [7] showed that much of the research focusing on social support has indicated an inverse association between social support and mental disorders. Sündermann et al., [8] also found that those who lack social support may have an increased risk of mental disorders and psychosis. Individuals in romantic relationships often report higher levels of overall life satisfaction and functioning [9]. However, the experience of emotional distress may impair one's ability to benefit from the protective effects of interpersonal relationships, specifically intimate, romantic connections. Therefore, understanding the impact that individual mental health may have on one's perceptions of satisfaction in a romantic relationship can be of importance. This is especially relevant given that these relationships seem to have a stabilizing effect on overall mental health [4].

Due to the known benefits of social support on a variety of mental health issues, a common recommendation to those experiencing certain types of psychological distress is to increase social contacts. However, for individuals with BPD navigating these social connections may be more tenuous. For example, in terms of close relationships, previous research has found that social networks for women with BPD were smaller than those for women without BPD. Women with BPD also self-reported that their social networks tended to be less supportive and satisfying [10]. According to Liebke et al., [11] constant worry about abandonment and loneliness are primary features of BPD. This type of anxious worrying may lead to poor interpersonal interactions. Therefore, for those with BPD increasing social connections may not provide the same protective effects, if the individual engages in behaviors which push people away or if their perceptions of the social support experienced is out of sync with the support received. Consequently, those who experience BPD-like symptoms may engage in behaviors and/or perceive the behaviors of others in such a manner that results in increased isolation within their social networks, thus reducing its protective benefits. 
When considering romantic relationships, these issues may be further compounded. Those with BPD report more perceptions of rejection and have fewer perceptions of acceptance [12]. Furthermore, those with BPD have little expectations of being socially accepted. They have difficulty adjusting their low expectations even after being given positive feedback. However, they do adjust their expectations, and lower them even more, when given negative feedback [11]. It should be clear that this type of negative orientation and feedback loop to social interactions could bring stress and tension to any interpersonal relationship, including intimate, romantic entanglements. Moreover, research has shown that while social support received from romantic relationships can be beneficial for mental health, it is perceived social support which most strongly predicts positive psychological adjustment [13]. Therefore, the protective mental health benefits provided by support in romantic relationships may be reduced, or eliminated, for those who endorse BPD-like patterns of emotional experiences. This can be due in part to a hyper-focus on negative information within the relationship and their sensitivity to rejection by their partner. Although the underlying mechanism through which this connection may occur has not been fully established empirically, research in the area confirms that those with BPD experience significant relationship problems which increases with the severity of the disorder [4].

Given what we know about the impact of clinical levels of BPD, it is of interest to see if a connection between poor interpersonal outcomes remains for those individuals who endorse BPD symptoms but are not treatment seeking. Thus, shedding additional light on the impact of the behaviors at a "sub-clinical" level. Moreover, for adolescents and young adults, psychological distress is of a growing concern. In 2017, young adults aged 18-25 years had the highest prevalence of a serious mental illness (SMI) ${ }^{2}$ at $7.5 \%$ compared to adults aged $26-$ 49 years $(5.6 \%)$ and aged 50 and older $(2.7 \%)$ (National Institute of Mental Health, 2019). Thus, examining BPD symptom endorsement in a cohort of young adults in dating relationships, may elucidate additional interpersonal risks for those in this age group.

For romantic relationships, it is also important to note that different people have different definitions for these connections. Monogamous relationships are considered the norm, especially within Western culture [14]. However, non-monogamous relationships are defined by a variety of different relationship arrangements, such as open relationships, and polyamory, where the parties involved agree that forming sexual or romantic bonds with others outside of the pair is acceptable $[15,16]$. Research suggests that non-monogamous relationships may be less satisfying than their counterpart [17]. Nonetheless, it is an empirical question as to whether those who experience BPD symptoms are affected by the types or status of relationships, as well as whether they have relationship experiences similar to those who do not endorse BPD symptoms. It remains important to explore specific aspects of the relationship dynamic to gain a deeper understanding of how a variety of relationship processes may connect to mental health experiences. Thus far, there is no research which explores features of relationships, like monogamy or sexual exclusivity, in concert with BPD symptoms.

In examining romantic relationships, commitment and monogamy may be considered pieces of the same puzzle. It has been demonstrated that individuals in committed relationships have greater well-being than those who are not $[18,19]$. Specifically, those who were in committed pre-marital relationships had fewer mental health problems and engaged in less problematic and risky behaviors. While there has been a distinction in research between married and non-married relationships [18], these connections can be further refined to note distinctions between those who are in serious dating relationships (committed, monogamous) in comparison to those who are in casual dating relationships (non-committed, non-monogamous). Thus, being in a committed, monogamous relationship versus a non-committed, non-monogamous relationship may potentially lead to differing effects when it comes to overall relationship satisfaction. This is confirmed by previous research on relationship uncertainty which found that individuals who are more certain about their relationships have higher levels of commitment to the relationship and engage in more behaviors to maintain the relationship [20]. Overall, a considerable amount of research links mental health to romantic relationship outcomes [2123]. Investigating whether there is a similar link between disorder symptoms when comparing individuals in committed, monogamous relationships versus individuals in non-committed, non-monogamous relationships can provide additional insight into interpersonal functioning.

The current study examined the association between BPD symptom endorsement and relationship satisfaction. Furthermore, we investigated whether there is a difference in BPD symptom endorsement (or severity) among those who are in committed, monogamous relationships compared to those in non-committed, non-monogamous relationships. Lastly, we explored the overall predictive contributions of BPD symptom endorsement and relationship type (i.e., committed, monogamous vs. non-committed, non-monogamous) to overall relationship satisfaction. Specifically, we hypothesized:

H1. BPD symptom endorsement is negatively associated with relationship satisfaction.

H2. Committed, monogamous relationships will have higher mean levels of relationship satisfaction, compared to non-committed, nonmonogamous relationships.

H3. Committed, monogamous relationships will have lower mean levels of BPD symptom endorsement, compared to non-committed, non-monogamous relationships.

\section{Materials and Method}

\section{Participants}

548 participants attending at a large, public southeastern university responded to study measures. A total of 433 participants responded to the relationship satisfaction measures and identified as being in either a committed, monogamous relationship $(n=343)$ or a noncommitted, non-monogamous relationship $(\mathrm{n}=90)$. Participants race/ ethnicity break down was $60.9 \%$ White, $13.4 \%$ Hispanic or Latino, $12.8 \%$ Black or African American, 6.9\% Asian, Native Hawaiian or Other Pacific Islander $0.2 \%, 4 \%$ other. Participant age ranged from 18 to $60(\mathrm{M}=21.5, \mathrm{SD}=5.7) .32 .8 \%$ of participants identified as Freshmen (1st year college students), 21.7\% were sophomores (2nd year college students), $23.7 \%$ were juniors (3rd year college students), $20.4 \%$ were seniors (4th year college students), and $0.4 \%$ were other (5th year+ college students or Graduate students). $67.5 \%$ of the participants identified as women and $31.6 \%$ were men.

\section{Measures}

\section{Relationship Status and Type}

Participants indicated their current relationship status as "yes" (scored as 1) for in a relationship or "no" (scored as 2) for not in a relationship. For those who were in a relationship, they also indicated if that relationship was "committed, monogamous" (scored as 1) or "non-committed, non-monogamous relationship" (scored as 0). Thus, relationship type was a dichotomous, categorical variable.

\section{Relationship Assessment Scale}

Participants completed the Relationship Assessment Scale (RAS) ${ }^{3}$ [2]. The Relationship Assessment Scale $(\alpha=0.89)$ is a 7 item selfreport assessment used to measure relationship satisfaction within an interpersonal relationship. The RAS uses a 5-point Likert response format with choices ranging from"unsatisfied" (1), to "extremely satisfied" (5). Total scores on the scale can range from 7 to 35 . Higher scale scores indicate higher levels of relationship satisfaction [2]. 


\section{Borderline Symptoms List}

Participants also completed the Borderline Symptoms List 23 $(\mathrm{BSL}-23)^{4}[1]$. The BSL-23 $(\alpha=0.96)$ is a 23 -item self-report measure that assesses symptoms of Borderline Personality Disorder (BPD). Each item presents a statement that many individuals with BPD may experience and coincides with the DSM-5 criteria for diagnosis, such as "I felt helpless" or "I felt worthless". Ratings for items vary from "not at all" (0), to "very strong" (4). The total score is calculated by dividing the total sum by the number of valid items and ranges from 0.0 to 4.0. Bohus et al., [1] explains that higher scores have been used to indicate increasing levels of severity of BPD symptoms. However, according to Kleindienst et al., [24] symptom severity for the total score should have a classification system. Thus, total scores can be further categorized as follows: none or low: $0-0.3$; mild: $0.3-0.7$; moderate: $0.7-1.7$; high: $1.7-2.7$; very high: $2.7-3.5$; and extremely high: 3.5-4. Thus, it is possible to examine BSL-23 scores as interval data or preset categories.

\section{Procedure}

Once institutional review board approval was secured, participants were able to access a link to study materials in Qualtrics, which is a web survey system. Participants were part of the General Psychology participant pool and could self-select to participate in the research for extra credit or research course credit. When the link was selected, a consent/informational sheet was provided which had to be viewed and individuals had to actively select "I agree to participate" to access the study measures. Individuals who selected "I do not agree to participate" were exited from the survey. Participants were asked about their current relationship status and then asked to complete the Relationship Satisfaction Scale [2], and the Borderline Symptoms List 23 [1]. Demographics items, such as age, race/ethnicity, sex, and college level were the final questions. Completion of study measures took approximately thirty minutes.

\section{Results}

\section{Descriptive Statistics. Borderline Symptom List (BSL-23).}

Participants' total scores on the BSL-23 ranged from 0 to 4 . Based on the proposed classification for the severity of BPD symptoms, the sample overall could be classified as "moderate" on symptom severity endorsement $(\mathrm{M}=0.71, \mathrm{SD}=0.75)$. The median total score was 0.43 . Thus, based on this metric which is less sensitive to outliers, the sample could still be classified as "mild" on BPD symptom severity [24].

Relationship Assessment Scale (RAS). Participants in romantic relationships reported reasonably high levels of relationship satisfaction, as measured in the RAS $(\mathrm{M}=25.93, \mathrm{SD}=6.538)$. These findings overall suggest that for this sample, levels of relationship satisfaction were high with lower levels of border line personality disorder symptoms.

\section{Hypothesis Testing.}

H1. BPD symptom endorsement is negatively associated with relationship satisfaction.

We hypothesized that as BPD symptom endorsement increased, relationship satisfaction would decrease. In previous examinations of BSL-23 scores, correlation and regression analyses have been used to establish validity and discriminate between clinical and non-clinical populations [1]. Thus, a bivariate Pearson correlation analysis was used to examine the association between relationship satisfaction and borderline personality symptoms. As predicted, there was a significant negative correlation between scores on the BSL-23 and RAS ( $\mathrm{r}=-.275, \mathrm{p}<.01$, one-tailed).

H2. Committed, monogamous relationships will have higher mean levels of relationship satisfaction, compared to non-committed, nonmonogamous relationships.

Individuals who self-identified as being in committed relationships were compared to individuals who self-reported as being in noncommitted relationships on relationship satisfaction. It was found that individuals in committed relationships $(\mathrm{M}=28.25, \mathrm{SD}=5.62)$ reported significantly higher relationship satisfaction than those in non-committed relationships $(\mathrm{M}=22.42, \mathrm{SD}=6.10)$. Thus, relationship satisfaction significantly varied by relationship type, $\mathrm{t}$ $(431)=8.58, \mathrm{p}<.01$.

H3. Committed, monogamous relationships will have lower mean levels of BPD symptom endorsement, compared to non-committed, non-monogamous relationships.

Individuals who self-identified as being in committed relationships were also compared to individuals in non-committed relationships on BPD symptom endorsement. It was found that individuals in committed relationships $(\mathrm{M}=0.67, \mathrm{SD}=0.72)$ reported similar levels of BPD symptom severity as those in non-committed relationships $(\mathrm{M}=0.63, \mathrm{SD}=0.67)$. Thus, BPD symptom endorsement did not significantly vary by relationship type, $\mathrm{t}(404)=0.45, \mathrm{p}=.65$.

\section{Exploratory Analyses}

Although not part of any specific hypotheses, we were interested in exploring the predictive impact of both relationship type (i.e., committed vs. non-committed) and borderline personality disorder symptom severity on overall relationship satisfaction. A sequential multiple regression analysis was completed with BPD symptom scores entered at the first step and relationship type entered at the second. As shown in Table 1, the addition of the variable "relationship type" results in a significant increase in R2 which suggests that relationship type provides additional variance explained in relationship satisfaction beyond what is explained by BPD symptom endorsement alone $(\mathrm{F}(2,403)=58.88, \mathrm{p}<.001)$.

\begin{tabular}{|c|c|c|c|c|c|c|c|c|}
\hline & & & \multicolumn{3}{|c|}{ Model 1} & \multicolumn{3}{|c|}{ Model 2} \\
\hline & $\mathrm{B}$ & SE B & $\beta$ & $\mathrm{p}$ & $\mathrm{B}$ & SE B & $\beta$ & $\mathrm{p}$ \\
\hline BPD Symptoms & -2.39 & 0.42 & -0.28 & $<.01$ & -2.47 & 0.38 & -0.28 & $<.01$ \\
\hline Relationship Type & --- & --- & --- & --- & 6.23 & 0.70 & 0.39 & $<.01$ \\
\hline $\mathrm{R} 2$ & \multicolumn{3}{|c|}{.08} & \multicolumn{5}{|c|}{.23} \\
\hline Sig. F for R2 $\Delta$ & \multicolumn{3}{|c|}{$<.001$} & & \multicolumn{4}{|c|}{$<.001$} \\
\hline
\end{tabular}

Table 1. Multiple RegressionCoefficients for Effects of BPD Symptoms and Relationship Type (Committed vs. NonCommitted) on Relationship Satisfaction

\section{Discussion}

The goal of the present study was to further explore an emerging area of research on borderline personality disorder symptom severity and relationship satisfaction in non-marital, romantic relationships. Interpersonal instability is a primary feature of those with borderline personality disorder [25]. Thus, we predicted that higher levels of borderline personality disorder symptom endorsement would lead to lower levels of relationship satisfaction. This hypothesis was supported. Those who reported more severe BPD symptom experiences also reported decreased relationship satisfaction. Previous research has shown the connection between BPD and poor relationship outcomes [4]. This finding indicates that even for those with "subclinical" levels of BPD symptoms, the emotional experiences, or 
behaviors, associated with the disorder can hurt relationship outcomes. The reasons for why this pattern emerges may be due to those high in borderline personality symptoms interpreting any negative interactions in their interpersonal relationships as rejection [12]. This sense of rejection may in turn lead to more hostile or sabotaging behaviors within the relationship.

For group differences between those in committed versus noncommitted relationships, we predicted that participants in committed, monogamous relationships would report higher levels of relationship satisfaction than those in non-committed relationships. Group mean comparisons supported this hypothesis. This pattern of results is in line with previous research on relationship uncertainty and commitment, which suggests that those individuals who are more certain and committed to their relationships overall, are happier with their relationships than those who are less certain [20]. Furthermore, we predicted that individuals in committed relationships would have lower BPD symptom endorsement levels than those who were in non-committed relationships. These data show that BPD total scores for those in committed relationships were slightly higher than the total scores for those in non-committed relationships. However, this difference was not significant. Therefore, our last hypothesis was not supported.

There are multiple possible explanations for this last result. First, this finding suggests that there may be no connection between relationship type and BPD symptom endorsement. Another possible explanation is related to the small size of the non-committed, nonmonogamous relationship group, which was under 100 participants. An estimate of power achieved for this comparison showed beta equal to .08 , which is too small to detect a small effect, if one is present. Therefore, the necessary power to detect an effect was not achieved for this comparison. Additional research should explore the connection between relationship type with a larger sample of individuals in non-committed, non-monogamous relationships.

Counter to our hypothesis, there was the trend towards higher BPD symptom endorsement in the committed relationship group. A possible explanation for this can be found in the characteristic behavior and emotional responses of those with BPD. Descriptions of the primary features of BPD suggest that unstable relationships are central to the disorder due to irrational emotional reactions, driven by fear of rejection, rather than a lack of desire for interpersonal connection [11]. Thus, it is possible that those who suffer from BPD symptoms would self-select into committed relationships rather than non-committed relationships. Engaging in casual, non-committed dating relationships may exasperate efforts to avoid abandonment and loneliness. Thus, individuals with mild to moderate levels of BPD may engage in relationship connections, which they perceive as "committed," that eventually erode under the pressure of the negative feedback loops and rejection sensitivity common to BPD sufferers. In this way these individuals may be "serial monogamists," who commit to relationships quickly, with a genuine desire for long-term connection. However, these relationships may also fizzle out quickly. Future research can more fully explore and explain this possible connection between relationship type and BPD symptoms if it exists.

Although we did not make any specific hypotheses about BPD symptoms and relationship type as predictors of relationship satisfaction, it was expected that both symptom severity and commitment in the relationship would be predictive of satisfaction. The regression analysis confirmed that in the prediction of relationship satisfaction, both BPD symptom severity and commitment provide significant explanatory power. Overall, BPD symptom severity and relationship type together explained $23 \%$ of the variance in relationship satisfaction. However, it is of note that relationship type (i.e., committed, or non-committed) provided most of the explanatory power of the variance explained. This result aligns with previous research which suggests that specific aspects of the relationship,or relationship functioning, are strong predictors for all participants regardless of BPD symptom endorsement. Thus, even for participants with low or no BPD symptoms, relationship commitment explains level of relationship satisfaction.

The current study presents some novel and interesting findings about BPD symptom severity and relationship functioning. No previous research has examined the impact of BPD symptoms, in conjunction with relationship commitment, on relationship satisfaction in nonmartial relationships. Thus, this research is noteworthy in its attempt to include relationship processes, like commitment, which are key to the navigation of romantic relationships. Prior research in the domain has mostly focused on the martial relationship and its effects on mental health. However, emerging research suggests that serious non-martial, dating relationships can have similar protective benefits [18]. This study takes the emerging literature a step further by demonstrating differences for those in serious dating relationships (i.e., committed) versus more casual dating relationships (noncommitted). Future research in this area can further explore sex differences between men and women on BPD symptom severity and relationship functioning. Thus, providing insight into how these variables may affect men and women differently.

There are also some of limitations of this work which must be considered and addressed in future research. These limitations include a non-clinical sample, the sample size for comparison of groups, and more robust measurement and analysis of relationship commitment. A goal of this research was to explore the impact of BPD symptoms at the sub-clinical level. Thus, the fact that our sample consisted of college students rather than a clinical sample may only be a limitation in generalizing the novel findings to a clinical population. Future researchers may want to explore these effects in a clinical sample of individuals with borderline personality disorder to replicate these findings with that population. Additionally, researchers may want to explore these constructs in a general population of non-college students. Though our sample was "sub-clinical" in that individuals were not in a treatment setting, we did find mild to moderate levels of BPD symptom severity. Thus, this sample may be more disordered than what would be seen in the general population.

The additional limitations are methodological weakness which can be addressed by future studies in the domain. Specifically, targeting data collection on a larger sample of individuals in casual dating, or non-committed, non-monogamous, relationships would allow for a better comparison of the effects of this status on relationship outcomes and BPD symptom severity. Additionally, a more robust measurement and analysis of relationship commitment and monogamy could provide a more detailed explanation of how this construct relates to the ways in which individuals with BPD symptoms navigate their relationships.

This research simply asked participants to self-identify as being in a committed, monogamous, or non-committed, non-monogamous relationship. Additional information about relationships should also be collected, such as length time in the relationship, number of previous relationships etc. Furthermore, a deeper examination of feelings of commitment to a relationship and partner were not explored. Although this information would still be self-report data, an expansion in the details connected to the type and manner of commitment may further our understanding of these relationship processes.

Stronger measures of experiences in monogamous vs. nonmonogamous relationships should also be explored. Recent research suggests that while committed monogamous relationships are still standard, people today are more open to committed yet polyamorous relationships [26]. Thus, an examination of whether these qualities of relationships have an impact on satisfaction and functioning for BPD symptom suffers could be of interest. Overall, future research in this area can also include more robust statistical analyses of more complex 
data which could incorporate all of the aforementioned components, such as hierarchical or multi-level modeling, thus providing more detailed and targeted information.

conclusion: The present study provides a foundation for continued exploration of specific features of relationships, such as commitment and monogamy, when considering the impact of BPD symptom severity on relationship satisfaction. Findings indicate that those high in borderline personality symptoms have lower levels of relationship satisfaction than those with less severe borderline personality symptoms. However, relationship commitment adds important predictive and explanatory power for relationship satisfaction as well. Overall, it is well established that romantic relationships provide protective benefits for mental health and functioning. Understanding the mechanisms through which we can improve relationship satisfaction may lead to the ability to take advantage of the stabilizing effects of interpersonal connections on mental health for those with BPD symptoms. Thus, if it is possible to repair or reduce the interpersonal dysfunction, which is a hallmark of BPD symptom severity, it may be possible to improve the mental health of those with BPD overall.

\section{Conflict of Interest (COI) Statement}

The authors have declared no conflict of interests.

\section{List of Abbreviations}

BPD Borderline Personality Disorder

BSL-23 Borderline Personality Symptom List 23

RASRelationship Assessment Scale

SMI Serious Mental Illness

\section{References}

1. Bohus, M., Kleindienst, N., Limberger, M. F., Stieglitz, R.D., Domsalla, M., Chapman, A. L., ... Wolf, M. (2009). The short version of the Borderline Symptom List (BSL-23): Development and initial data on psychometric properties. Psychopathology, 42(1), 32-39. https://doi-org.ezproxy.net.ucf. edu/10.1159/000173701

2. Hendrick, S. S., Dicke, A., \& Hendrick, C. (1998). The Relationship Assessment Scale. Journal of Social and Personal Relationships, 15(1), 137-142. https://doi-org.ezproxy.net.ucf. edu/10.1177/0265407598151009

3. Grant, B. F., Chou, S. P., Goldstein, R. B., Huang, B., Stinson, F. S., Saha, T. D., ... Ruan, W. J. (2008). Prevalence, correlates, disability, and comorbidity of DSM-IV borderline personality disorder: Results from the Wave 2 National Epidemiologic Survey on Alcohol and Related Conditions. The Journal of Clinical Psychiatry, 69(4), 533-545. https://doi-org.ezproxy. net.ucf.edu/10.4088/JCP.v69n0404

4. Kuhlken, K., Robertson, C., Benson, J., \& Nelson-Gray, R. (2014). The interaction of borderline personality disorder symptoms and relationship satisfaction in predicting affect. Personality Disorders: Theory, Research, and Treatment, 5(1), 20-25. https://doi-org.ezproxy.net.ucf.edu/10.1037/per0000013

5. Hill, J., Pilkonis, P., Morse, J., Feske, U., Reynolds, S., Hope, H.,... Broyden, N. (2008). Social domain dysfunction and disorganization in borderline personality disorder. Psychological Medicine, 38, 135-146. doi:10.1017/S0033291707001626

6. Umberson Debra, \& Montez Jennifer Karas. (2010). Social Relationships and Health: A Flashpoint for Health Policy. Journal of Health and Social Behavior, 51, S54-S66.

7. Henderson, A. S. (1992). Social support and depression. In H. O. F. Veil \& U. Baumann (Eds.), Meaning and measurement of social support. New York, NY: Hemisphere.
8. Sündermann, O., Onwumere, J., Kane, F., Morgan, C., \& Kuipers, E. (2014). Social networks and support in first-episode psychosis: Exploring the role of loneliness and anxiety. Social Psychiatry and Psychiatric Epidemiology, 49, 359 -366. http:// dx.doi.org/10.1007/s00127-013- 0754-3

9. Porter, D. \& Chambless, E. (2017). Social Anxiety and Social Support in Romantic Relationships. Behavior Therapy Volume 48, Issue 3, May 2017, Pages 335-348 https://doi.org/10.1016/j. beth.2016.12.002

10. Lazarus, S. A., \& Cheavens, J. S. (2017). An examination of social network quality and composition in women with and without borderline personality disorder. Personality Disorders: Theory, Research, And Treatment, 8(4), 340-348. doi:10.1037/ per0000201

11. Liebke, L., Koppe, G., Bungert, M., Thome, J., Hauschild, S., Defiebre, N., ... Lis, S. (2018). Difficulties with being socially accepted: An experimental study in borderline personality disorder. Journal of Abnormal Psychology, 127(7), 670-682. https://doi-org.ezproxy.net.ucf.edu/10.1037/abn0000373.supp (Supplemental)

12. Lazarus, S. A., Scott, L. N., Beeney, J. E., Wright, A. C., Stepp, S. D., \& Pilkonis, P. A. (2018). Borderline personality disorder symptoms and affective responding to perceptions of rejection and acceptance from romantic versus nonromantic partners. Personality Disorders: Theory, Research, And Treatment, 9(3), 197-206. doi:10.1037/per0000289

13. Prati, G.\&Pietrantoni, L. (2010). The relation of perceived and received social support to mental health among first responders: A meta-analytic review Journal of Community Psychology, 38 (3) pp. 403-417, 10.1002/jcop.20371

14. Wood, J., Desmarais, S., Burleigh, T., \& Milhausen, R. (2018). Reasons for sex and relational outcomes in consensually nonmonogamous and monogamous relationships: A selfdetermination theory approach. Journal of Social and Personal Relationships, 35(4), 632-654. https://doi-org.ezproxy.net.ucf. edu/10.1177/0265407517743082

15. Haupert, M. L., Gesselman, A. N., Moors, A. C., Fisher, H. E., \& Garcia, J. R. (2017). Prevalence of experiences with consensual nonmonogamous relationships: Findings from two national samples of single Americans. Journal of Sex and Marital Therapy, 43(5), 424-440.

16. Mogilski, J. K., Reeve, S. D., Nicolas, S. C. A., Donaldson, S. H., Mitchell, V. E., \& Welling, L. L. M. (2019). Jealousy, consent, and compersion within monogamous and consensually non-monogamous romantic relationships. Archives of Sexual Behavior. https://doi-org.ezproxy.net.ucf.edu/10.1007/s10508018-1286-4

17. Levine, E. C., Herbenick, D., Martinez, O., Fu, T.-C., \& Dodge, B. (2018). Open relationships, nonconsensual nonmonogamy, and monogamy among US Adults: Findings from the 2012 national survey of sexual health and behavior. Archives of Sexual Behavior. https://doi-org.ezproxy.net.ucf.edu/10.1007/ s10508-018-1178-7

18. Braithwaite, S. R., Delevi, R., \& Fincham, F. D. (2010). Romantic relationships and the physical and mental health of college students. Personal Relationships, 17(1), 1-12. https:// doi.org/10.1111/j.1475-6811.2010.01248.x

19. Selby, E. A., Braithwaite, S. R., Joiner, T. E., Jr., \& Fincham, F. D. (2008). Features of borderline personality disorder, perceived childhood emotional invalidation, and dysfunction within current romantic relationships. Journal of Family Psychology, 22(6), 885-893. https://doi.org/10.1037/a0013673 
20. Knobloch , L. K. , \& Solomon , D. H. ( 2002 ). Information seeking beyond initial interaction: Negotiating relational uncertainty within close relationships. Human Communication Research , 28, 243-257.

21. Edwards-Stewart, A., Rennebohm, S. B., DeSimone, J., Willey, B., Smolenski, D. J., \& Hoyt, T. (2018). Relationship satisfaction and mental health treatment among active-duty military. Couple and Family Psychology: Research and Practice, 7(3-4), 201211. https://doi-org.ezproxy.net.ucf.edu/10.1037/cfp0000108

22. Whisman, M. A. (1999). Marital dissatisfaction and psychiatric disorders: Results from the National Comorbidity Survey. Journal of Abnormal Psychology, 108. 701-706.

23. Whisman, M. A., Sheldon, C. T., \& Goering, P. (2000). Psychiatric disorders and dissatisfaction with social relationships: Does type of relationship matter? Journal of Abnormal Psychology, 109(4), 803-808. https://doi-org. ezproxy.net.ucf.edu/10.1037/0021-843X.109.4.803
24. Kleindienst, N., Jungkunz, M. \& Bohus, M. (2020). A proposed severity classification of borderline symptoms using the borderline symptom list (BSL-23). Borderline Personality Disorder and Emotion Dysregulation, 7:11. https://doi. org/10.1186/s40479-020-00126-6

25. Lazarus, S. A., Beeney, J. E., Howard, K. P., Strunk, D. R., Pilkonis, P. A., \& Cheavens, J. S. (2019). Characterization of relationship instability in women with borderline personality disorder: A social network analysis. Personality Disorders: Theory, Research, and Treatment. https://doi.org/10.1037/ per0000380

26. Loue, S. (2006). Multi-bonding: Polygamy, polygyny, polyamory. In S. Loue (Ed.), Sexual partnering, sexual practices, and health (pp. 27-53). New York: Springer. 